\title{
DTL facilitates the Fanconi anemia pathway for ultraviolet-induced DNA repair in retinal pigment epithelial cells
}

\author{
JiUchun GUO ${ }^{1}$; Jie PAN ${ }^{2, *}$; QIANQIAN GUO ${ }^{2}$ \\ 1 Binzhou Second People's Hospital, Binzhou, 256800, China \\ 2 Department of Ophthalmology, Zibo Central Hospital, Zibo, 255000, China
}

Key words: DTL, Fanconi anemia pathway, Retinal pigment epithelial, p53

\begin{abstract}
The excessive energy of light, especially the invisible rays with lower wavelength, is basically absorbed by retinal pigment epithelium (RPE) and usually causes DNA damage. The molecular mechanism behind DNA damage repair response to this frequent stress in RPE is not clearly understood. In this study, we determined that the Fanconi anemia (FA) pathway was activated in human RPE ARPE-19 cells after ultraviolet (UV) B and C treatment. Moreover, immunoprecipitation (IP) of FANCD2 indicated that denticleless E3 ubiquitin protein ligase homolog (DTL) closely interacted with FANCD2. Knockdown of DTL weakened the activity of the FA pathway in ARPE-19 cells responding to UV treatment. Finally, the DTL promoter was incubated with a biotin-labeled probe and pulled down by streptavidin beads followed by the genomic DNA sonication. p53 was indicated by mass spectrum and further determined by chromatin IP assay. Taken together, our results demonstrated that DTL regulated by p53 could activate the FA pathway for UV-induced DNA damage repair in retinal pigment epithelial cells.
\end{abstract}

\section{Introduction}

In the normal visible light or sunshine, only a small part reaching the retina is attributed to the sense of sight, while the rest of it is usually absorbed by the retinal pigment epithelium (RPE). RPE is subjected to greater energy from the light with lower wavelengths, which ultimately causes adverse effects on the photoreceptors and vision (Patton et al., 1999). Ultraviolet (UV) radiation whose spectrum less than $400 \mathrm{~nm}$ can be further divided into three subtypes UVA (315-400 nm), UVB (280-315 nm and UVC $(100-280 \mathrm{~nm})$. The irradiation of UVB and UVC is directly deleterious to DNA through photochemical modification. The covalently bonded pyrimidine dimers are generated by UV-derived photons and cause the DNA strand twist to interfere with the process of DNA replication and transcription (Roy, 2017). Besides, DNA double-strand breaks (DSBs) and interstrand crosslinking (ICL) can also be induced by UV lesions (Han et al., 2015; Yin et al., 2017).

Fanconi anemia (FA) signaling pathway plays a crucial role in repairing DSBs and ICL. ICL usually happens when replication forks encounter DNA lesions. Removal of ICL is generally performed by the FA pathway, especially the core

*Address correspondence to: Jie Pan, panjie198204@163.com Received: 13 January 2021; Accepted: 07 February 2021 complex of FANCD2-FANCI and other multiple enzymes for DNA repair. Concurrently, cutting DNA lesions and untying ICL by these enzymes will usually generate DSBs, and monoubiquitinated FANCD2 can trigger translesion.

DNA synthesis (TLS), homologous recombination repair (HRR), and nucleotide excision repair (NER) for DSBs restoration (Federico et al., 2016). The FA pathway has been widely studied in protecting from UV-induced, or spontaneous, DNA damage in various cell/tissue types and diseases, however, seldom in RPE.

In this study, human RPE ARPE-19 cells were exposed to UVB light, and we investigated the activity of the FA pathway and further revealed the regulatory factors for FA pathway activation. Our study can provide evidence for a novel regulatory mechanism on UV-induced DNA damage response in RPE.

\section{Materials and Methods}

\section{Cell culture}

RPE cell line (ARPE-19) obtained from American type culture collection (ATCC, Manassas, VA, USA) were cultured in DMEM medium supplemented with $10 \%$ fetal bovine serum (Thermo Fisher Scientific, Waltham, MA, USA). ARPE-19 cells were maintained in a $37^{\circ} \mathrm{C}$ incubator with $5 \% \mathrm{CO}_{2}$. For $\mathrm{UV}$ exposure, the medium was removed initially, then UVB 
$\left(25 \mathrm{~mJ} / \mathrm{cm}^{2}\right)$ were performed using narrow-band UVB light (Sunfine, Zhejiang, China) for 5, 10, 20, and $40 \mathrm{~s}$ (Panneerselvam et al., 2016), and cells were immediately added fresh medium for 4 h-culture. The siRNA oligonucleotides of DTL (5'-CAAUGGACACCAGAACUCUACCUUU-3') (Jin et al., 2006) and FANCI (5'CACGGGCAUCUGGGAGAUAUA-3') (Cheung et al., 2017) synthesized from Genepharma (Shanghai, China) were transfected into ARPE-19 cells using lipo RNAiMAX (Thermo Fisher Scientific) according to the manufacturer's instruction. After $48 \mathrm{~h}$ transfection, cells were harvested for the next experiments. Taqman DNA probe with labeled biotin (Thermo Fisher Scientific) for DTL promoter was synthesized as the following location: GRCh38: chr1: 212034692-212034967 containing two putative p53 binding sites predicted by JASPAR database (Fornes et al., 2020).

\section{Immunofluorescence staining assay}

ARPE-19 cells were fixed within a $4 \%$ paraformaldehyde solution and washed by phosphate buffer saline (PBS). Then, cells were permeabilized with $0.1 \%$ Triton-X 100 (EMD Millipore, Billerica, MA, USA) and blocked with $0.5 \%$ horse serum in PBS. Immunostainings of samples were performed using the antibodies of $\gamma \mathrm{H} 2 \mathrm{AX}$ (1:200, Cell Signaling Technology, Beverly, MA, USA) and FANCD2 (1:200, Abcam, Cambridge, MA, USA) overnight at $4^{\circ} \mathrm{C}$. After washing with PBS four times (5 min per time), the appropriate secondary antibodies (1:20000, Jackson ImmunoResearch, West Grove, PA, USA) were used to incubate for $2 \mathrm{~h}$ at room temperature, following the further incubation with DAPI for $15 \mathrm{~min}$, washed with PBS four times. After drying in the air, slices were dropped with a mounting medium (Thermo Fisher Scientific) and coated with cover glasses. The positive staining was statistically analyzed using Image J.

\section{TUNEL assay}

TUNEL assay was performed using One Step TUNEL Apoptosis Assay Kit (Beyotime Biotechnology, Shanghai, China). dUTP stained by Cyanine 3 was added at the 3'-OH by Terminal deoxynucleotidyl transferase. The experimental procedure was followed by the manufacturer's instructions.

\section{Western blot (WB) assay}

$1 \times 10^{6}$ cells were added $500 \mu \mathrm{L}$ RIPA buffer (Thermo Fisher Scientific) with protease inhibitor cocktail (Beyotime Biotechnology) and quantified the concentration using BCA methods. Aliquots of proteins $(40 \mu \mathrm{g})$ were added into the lanes of $10 \%$ SDS polyacrylamide gel (for detection of FANCD2 monoubiquitination, 7.5\% SDS gel was prepared), and the proteins were separated through electrophoresis and transferred onto nitrocellulose membranes. Subsequently, the membranes were congested with $5 \%$ nonfat dry milk in $0.01 \mathrm{M}$ PBS buffer ( $\mathrm{pH} 7.4$ ) and $0.05 \%$ Tween-20 for $1 \mathrm{~h}$ at room temperature (RT). The blocked membranes were then incubated with primary antibodies of FANCD2 (1:1500, Abcam), FANCI (1:2000, Novus, Centennial, CO, USA), $\gamma \mathrm{H} 2 \mathrm{AX}$ (1:2000, Cell Signaling Technology, Beverly, MA, USA), H2AX (1:2000, Cell Signaling Technology), DTL (1:2000, Novus), CDT1 (1:2000, Cell Signaling Technology) and $\beta$-actin (1:5000, Beyotime Biotechnology) overnight at $4^{\circ} \mathrm{C}$, followed by incubation with the appropriate secondary antibodies (horseradish peroxidase-conjugated rabbit antimouse diluted 1:10000 and donkey anti-rabbit diluted 1:5000, Biotechnology) for $30 \mathrm{~min}$ at RT. The expression was determined by the enhanced chemiluminescence method using Amersham Imager 600 system (GE Healthcare Life Sciences, Pittsburgh, PA, USA), whereas the density of the immunoblots was measured with Quantity One 4.62 software (Bio-Rad Laboratories, Hercules, CA, USA).

\section{Quantitative polymerase chain reaction ( $q P C R)$ assay}

DNA templates taken from ChIP were conducted via qPCR using the QuantStudio 3 system (Thermo Fisher Scientific). In accordance with the given instructions, $95^{\circ} \mathrm{C}$ for $30 \mathrm{~s}$ for initial denaturation, followed by 40 cycles at $95^{\circ} \mathrm{C}$ for $5 \mathrm{~s}$, appropriate annealing temperatures of $10 \mathrm{~s}$ and $72^{\circ} \mathrm{C}$, then $30 \mathrm{~s}$ were set up for PCR conditions. Ct values were harvested and calculated by using the delta-delta method. Primer sequences of DTL promoter for ChIP assay are listed as follows: P1 F: 5'-CAGCTTCCCAAAGTGCGGGGATT-3', R: 5'-GACCTCCTCAATCAAGTGGAAGAAC-3'; P2: 5'TTGTGGTTTTTTTCTTACAACTGCAAAA-3', R: 5'-TGTGTTTTTCAAACACTTTCCTCCTCATT-3'; P3: F: 5'-GGTACTGTACCCACTTTGCAGAC-3', R: 5'-AATAGTAATCTTGCACCGACTTGAC-3'; P4: 5'-TCGAATACCTTTGTCCAATTCCATAA-3', R: AGGGCTTGACAGCGCGCGAAG-3’.

\section{Immunoprecipitation (IP) assay}

Briefly, $5 \times 10^{6}$ cells were fixed with $1 \%$ formaldehyde, quenched with $0.125 \mathrm{M}$ glycine at room temperature, and then lysed in $500 \mu \mathrm{L}$ of lysis buffer $(10 \mathrm{mM}$ Tris- $\mathrm{HCl}$; $\mathrm{pH}$ 8.0), $10 \mathrm{mM} \mathrm{NaCl}$, and 0.2\% IGEPAL CA-630 (Thermo Fisher Scientific)) on ice for $30 \mathrm{~min}$. The genomic DNA was sonicated into 200-500 bp. Ten percent of each whole-cell lysate was stored as input, and the rest of the lysate was incubated with $1 \mu \mathrm{g}$ of the primary antibodies of p53 or IgG (Abcam) at $4^{\circ} \mathrm{C}$ overnight. Then, an additional 2-h pulldown was performed at $4^{\circ} \mathrm{C}$ with protein-A beads (Thermo Fisher Scientific). For chromatin IP, DNA was purified from beads by phenol-chloroform. Primers designed to encompass approximately $150 \mathrm{bp}$ around the target regions were used to detect the enrichment of p53 using qPCR assay. For regular IP, beads added $20 \mu \mathrm{L}$ lysis buffer and $5 \mu \mathrm{L} 5 \times$ loading buffer were boiled at $100^{\circ} \mathrm{C}$ for $10 \mathrm{~min}$. The protein interaction was investigated by WB. Mass spectrum services were provided by the Shandong Analysis and Test Center (Jinan, Shandong, China).

\section{Firefly luciferase reporter assay}

Different fragments (P1-4) of DTL promoter were cloned respectively and inserted into a pGL3-Basic vector (Addgene, Watertown, MA, USA) between HindIII and XhoI sites. The sequences of two putative p53 binding sites ("GACAAGCCGAGGCTAGAT" and "GTCAAGTCGGTGCAAGAT”) were deleted respectively as the mutant P3 fragments. $1 \mu \mathrm{g}$ vectors were transfected into ARPE-19 cells for $48 \mathrm{~h}$ followed by UVB treatment, and the luciferase relative intensity was detected by Multiskan FC microplate reader (Thermo Fisher Scientific). 


\section{Statistical analysis}

The data were presented as means \pm SEM. All statistical analyses were performed using SPSS 20.0 statistics software. Group comparisons were analyzed with one-way ANOVA. A $p$-value less than 0.05 was considered as a significant difference.

\section{Results}

The activation of FA pathway responding to $U V$-induced DNA damage in ARPE-19 cells

ARPE- 19 cells were treated with UVB $\left(25 \mathrm{~mJ} / \mathrm{cm}^{2}\right)$ for different periods of 5, 10, 20, and $40 \mathrm{~s}$. After $4 \mathrm{~h}$ self-recovery, high levels of $\gamma \mathrm{H} 2 \mathrm{AX}$ due to Ser 139 phosphorylation of H2AX induced by DNA damage were detected in $10 \mathrm{~s}$ and $20 \mathrm{~s}$ of UVB (Figs. 1A1B). The enrichment of FANCD2 was highly overlapped with foci in $10 \mathrm{~s}$ and $20 \mathrm{~s}$ of UVB compared to the other conditions (Fig. 1A). TUNEL assay indicated that cell apoptosis extensively occurred in ARPE-19 cells with UVB treatment for $40 \mathrm{~s}$ (Fig. 1C). Herein, we determined that cells were completely recovered under $5 \mathrm{~s}$ exposure of UVB and were hard to observe DNA repair after $4 \mathrm{~h}$. On the contrary, UV-induced DNA damage failed to be fixed by the selfrepairing system and caused cell death under the condition of $40 \mathrm{~s}$. We provided appropriate timing conditions of UVB treatment for DNA damage repairing study in ARPE-19 cells.

FANCI was knockdown in ARPE-19 cells, and cells were further treated with $20 \mathrm{~s}$-UVB and $4 \mathrm{~h}$ self-recovery. We observed that DNA damage was more serious in FANCI knockdown (Fig. 1A), and the expression of $\gamma \mathrm{H} 2 \mathrm{AX}$ was higher while monoubiquitination of FANCD2 was reduced in FANCI knockdown compared to control and wild type ARPE-19 cells (Figs. 1A, 1B). Cell death was substantially apparent in FANCI knockdown compared to the other conditions (Fig. 1C). Taken together, the FA pathway indeed played an essential for UV-induced DNA damage repair in ARPE-19 cells.

\section{DTL was essential for FANCD2 monoubiquitination}

To further investigate the regulatory mechanism behind the activation of the FA signaling pathway in RPE, FANCD2 IP
A

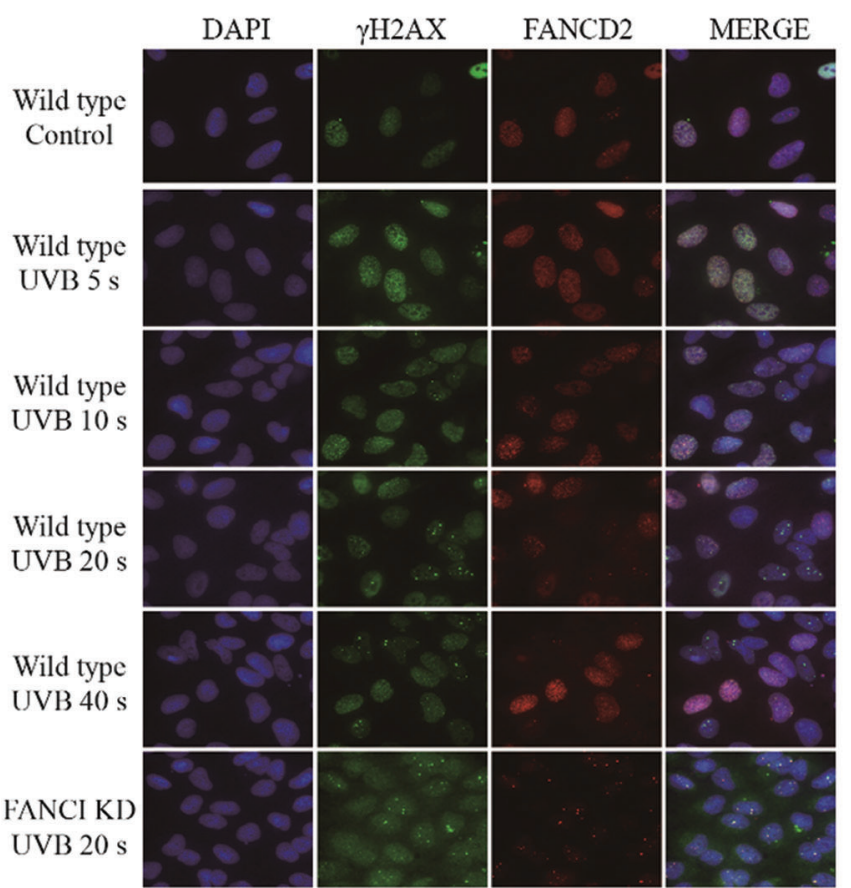

B

Wild type Wild type Wild type Wild type Wild type FANCI KD Control UVB $5 \mathrm{~s}$ UVB $10 \mathrm{~s}$ UVB $20 \mathrm{~s}$ UVB $40 \mathrm{~s}$ UVB $20 \mathrm{~s}$

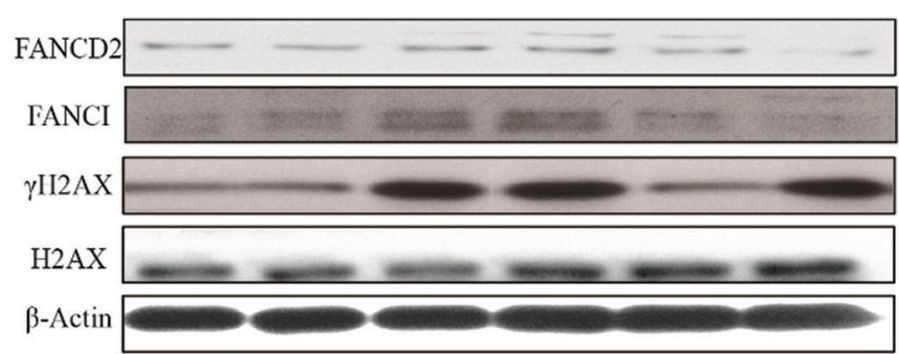

$\mathrm{C}$ Wild type
Control

Wild type UVB $5 \mathrm{~s}$

\section{Wild type} UVB $10 \mathrm{~s}$

Wild type UVB $20 \mathrm{~s}$

Wild type UVB $40 \mathrm{~s}$

\section{FANCI KD} UVB $20 \mathrm{~s}$

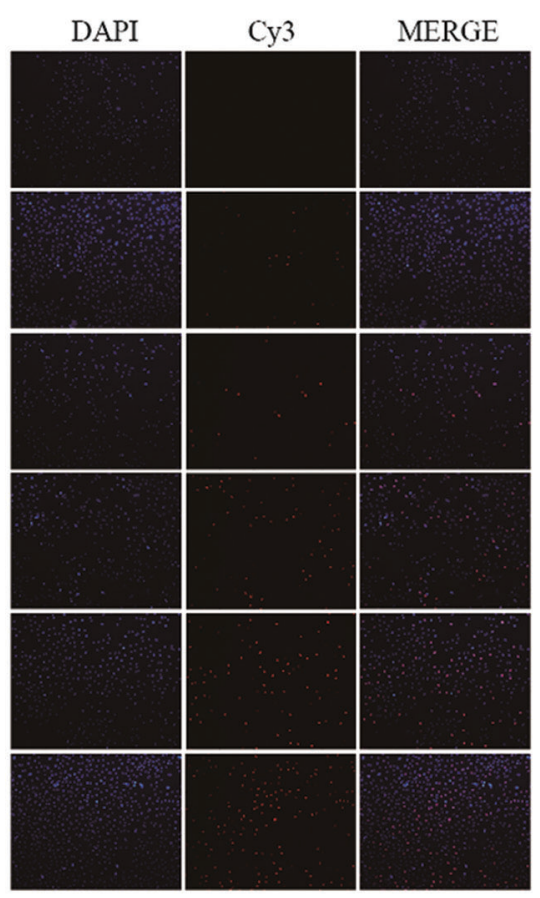

FIGURE 1. UVB-induced DNA damage in ARPE-19 cells.

The staining (A) and the expression (B) of FANCD2 and $\gamma \mathrm{H} 2 \mathrm{AX}$ in ARPE-19 cells under the different conditions of UVB exposure detected by IF and WB assay. The density of bright foci indicates the DNA damage severity. The cell apoptosis in ARPE-19 cells responding to different conditions of UVB exposure, detected by TUNEL assay (C). Positive staining indicates the cell apoptosis levels. The data are presented as the mean \pm standard error of the mean of ten individual vision fields. "*” represents the comparison to wild type $20 \mathrm{~s}$ with a $p$-value less than 0.05 by one-way ANOVA.

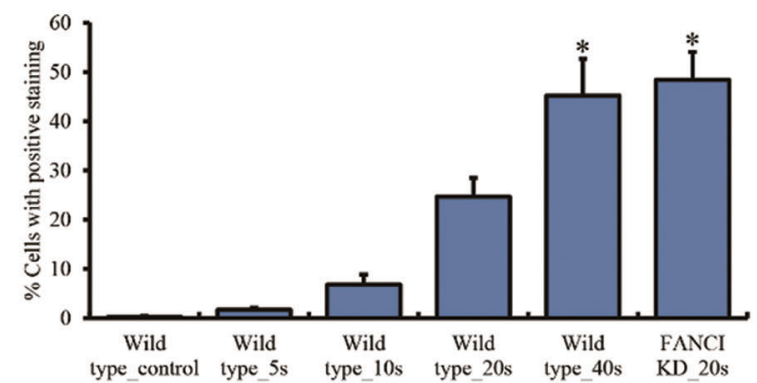


was performed in wild type and FANCI knockdown in ARPE19 cells with $20 \mathrm{~s}$-UVB treatment. The band at $65 \mathrm{kD}$ in wild type ARPE-19 cells was picked up for mass spectrum (Fig. 2A). Human denticleless E3 ubiquitin protein ligase homolog (DTL) as the candidate binding protein with FANCD2 was verified by reverse IP study. We confirmed that DTL was substantially interacted with FANCD2 in wild type but not FANCI knockdown ARPE-19 cells with UV treatment (Figs. 2B, 2C). Furthermore, DTL was highly expressed upon UV exposure, but knockdown of DTL could weaken but not completely destroy the FANCD2 or FANCI monoubiquitination after UVB treatment in ARPE-19 cells (Fig. 2D). Consistently, the expression of CDT1, which was the target of the DTL-mediated E3 ligase complex, was remarkably compromised by UVB treatment and rescued after DTL knockdown. These results above indicated that DTL at upstream might have an important effect on FA pathway activation upon UV-induced damage.

Transcription activity of DTL was enhanced by p53 in UV treated ARPE-19 cells

Next, we studied the mechanism of DTL transcription regulated by UVB. DTL promoter was incubated with biotin-labeled probe and pulled down using streptavidin beads after genomic DNA sonication. Confirming that DTL promoter was pulled down successfully (Fig. 3A), one band at $50 \mathrm{kD}$ around was especially there in wild type APRE-19 cells with UV treatment compared to control (Fig. 3B), and p53 was identified by mass spectrum. ChIP-qPCR was used to confirm that p53 enrichment on the promoter of DTL was higher in UV treatment compared to control. The fragment detected by primers P3 contained two putative p53 binding sites predicted by JASPAR compared to P1, P2, and $\mathrm{P} 4$, and was shown the substantial p53 enrichment upon UV treatment in ARPE-19 cells (Fig. 3C). Consistently, the fragments of $\mathrm{P} 1, \mathrm{P} 3$, and $\mathrm{P} 4$ individually indeed contributed to DTL transcription activation by firefly luciferase reporter assay, and P3 was the only p53 binding site that effectively responded to UV stimulation (Fig. 3D). Moreover, two p53 motifs in P3 were deleted, and the transcription activities were nearly completely lost (Fig. 3E), which also determined the binding ability of p53 on the DTL promoter.

Collectively, our results suggested that DTL promoted by p53 could enhance the activity of the FA pathway responding to UV-induced DNA damage in RPE cells.

\section{Discussion}

DNA replication initiation in eukaryotic organisms is highly conserved regarding the DNA licensing factors, including the origin of replication complex, mini-chromosome maintenance complex, chromatin licensing and DNA replication factor 1 (CDT1), and other proteins. CDT1 contributes to preventing DNA replication initiation in $G$ and $M$ phases to guarantee only once DNA replication in a given cell cycle (Blow and Dutta, 2005). In the $S$ phase, CDT1 was recognized by DTL and further degraded by the E3 ubiquitin ligase complex of CUL4-DDB1-DTL. Following DNA damage by UV irradiation, CDT1 degradation on chromatin was similarly modulated by the complex of

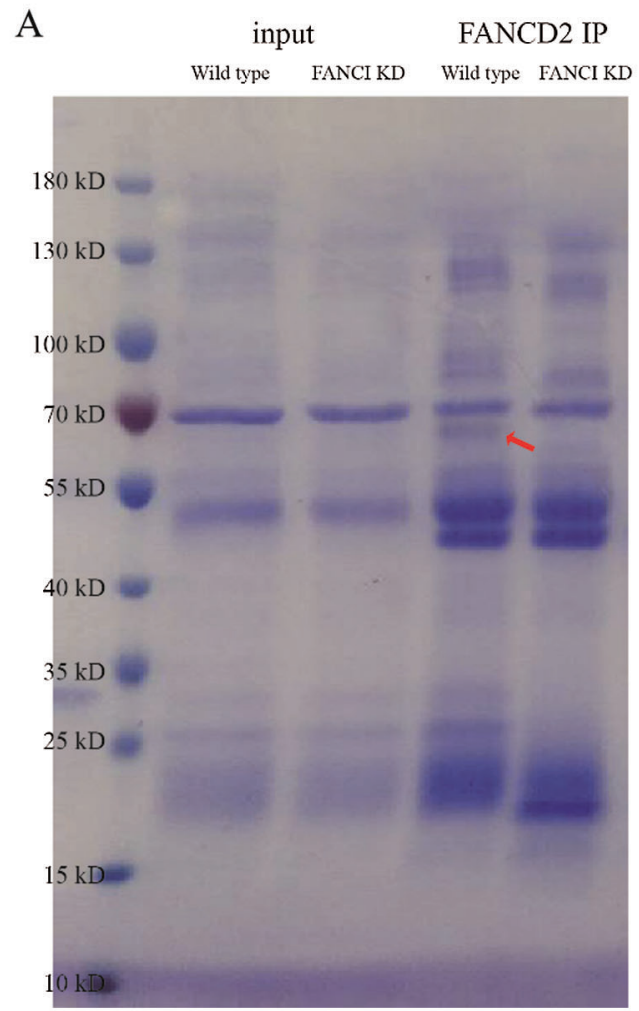

B

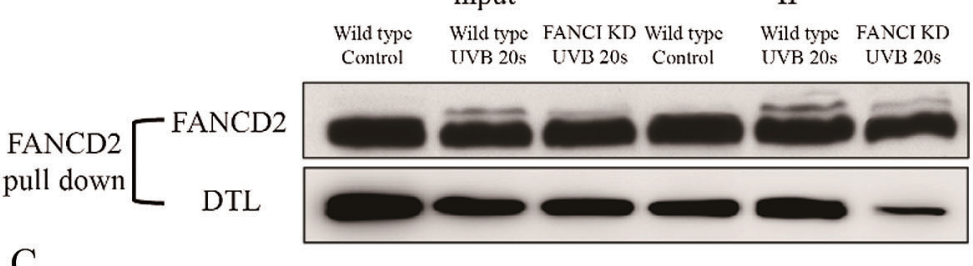

$\mathrm{C}$

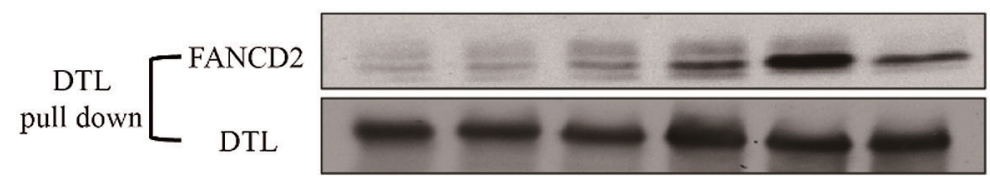

$\mathrm{D}$

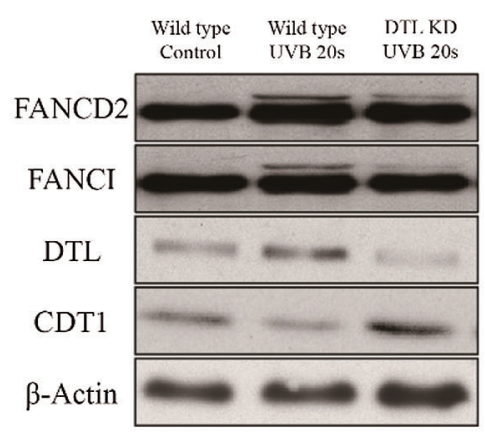

FIGURE 2. FA pathway activation facilitated by DTL under UV exposure.

Coomassie brilliant blue staining shows DTL pulled down by FANCD2 in wild type ARPE-19 cells compared to FANCI knockdown highlighted by the red arrow (A). Reverse IP indicates the interaction between FANCD2 (B) and DTL (C). FA pathway activation impacted by DTL knockdown in ARPE-19 cells exposed by UV irradiation (D). 

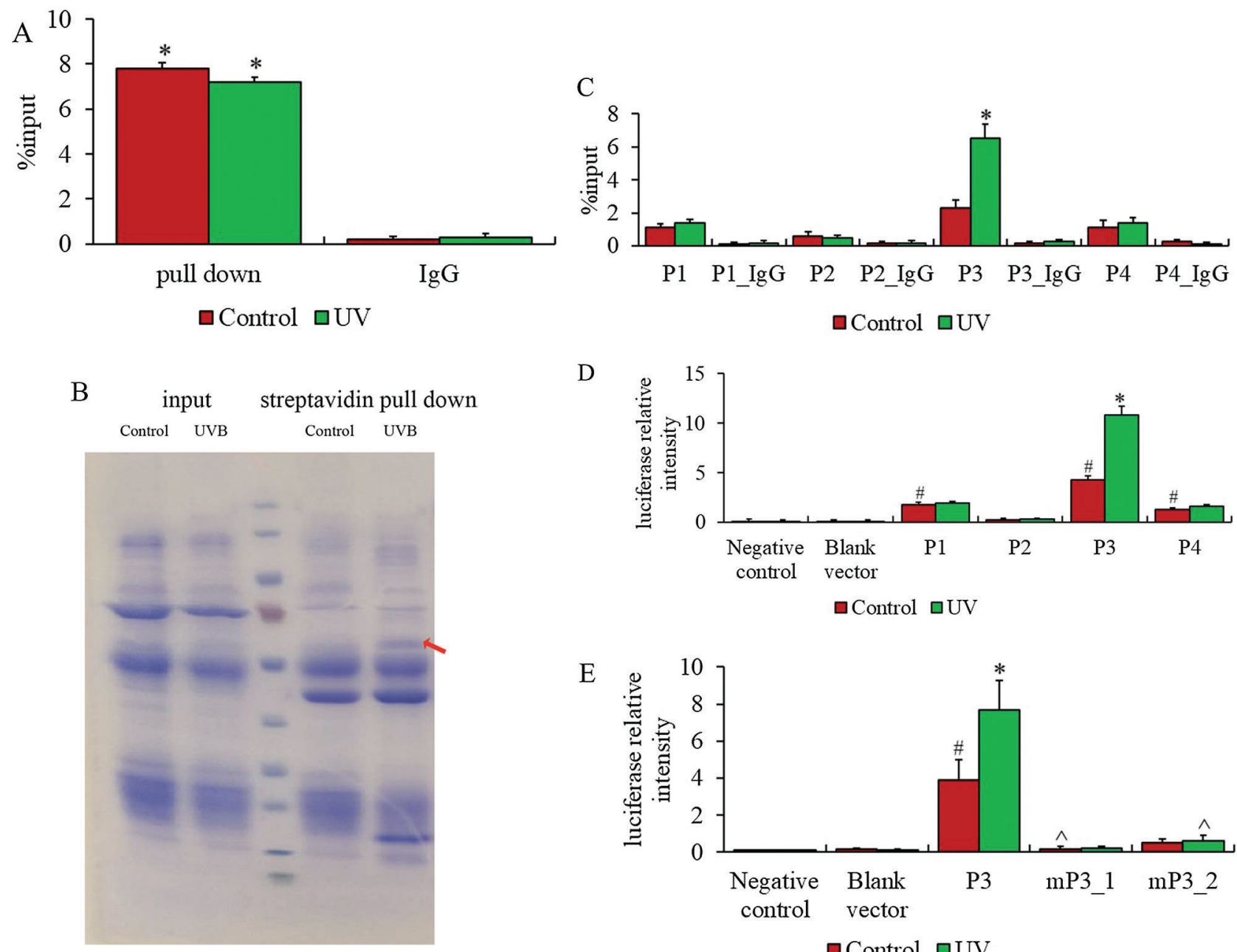

$\mathrm{D}$

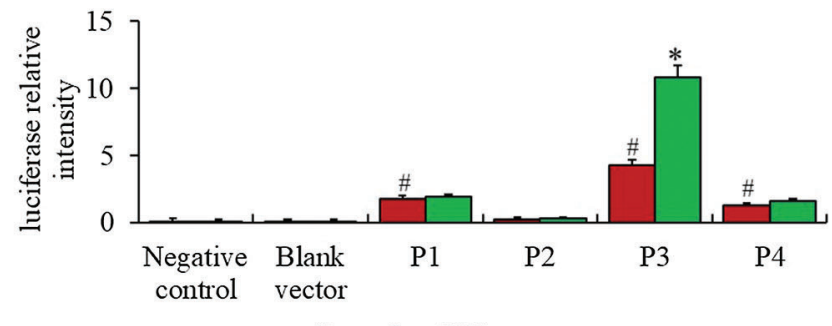

aControl aUV

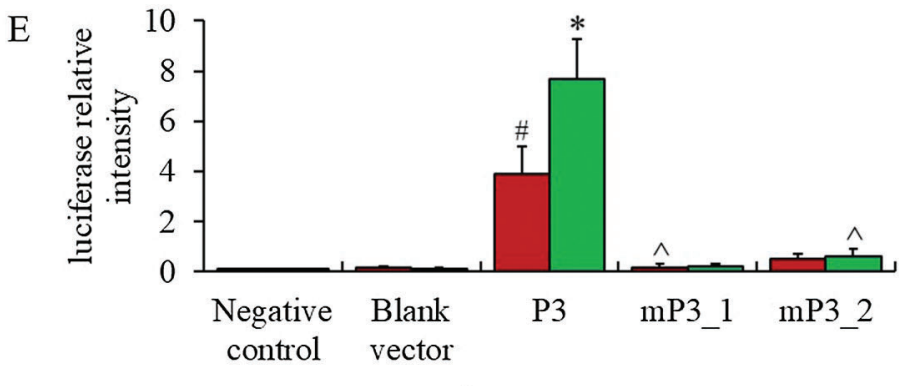

FIGURE 3. DTL transcription regulated by $\mathrm{p} 53$ in UV response.

The promoter of DTL in pull-down products was detected by qPCR (A). Coomassie brilliant blue staining shows p53 pulled down by biotinlabeled probe for DTL promoter in UV treatment compared to control highlighted by the red arrow (B). The enrichment of p53 at DTL promoter, detected by ChIP-qPCR assay (C). The transcription activities of putative p53 binding sites of DTL promoter detected by firefly luciferase reporter assay (D). The transcription activities of DTL promoter with two putative p53 binding sites deletion within P3 (E). Negative control means ARPE-19 cells without transfection. Blank vector means APRE-19 cells transfected by a blank pGL3-Basic vector. P1-4 represents APRE-19 cells transfected by the pGL3-Basic vector with four respective fragments of DTL promoter. P3 contains two putative p53 binding sites predicted by JASPAR. mP3_1 and mP3_2 mean APRE-19 cells transfected by the pGL3-Basic vector with the deletion of these two p53 binding sites. The data are presented as the mean \pm standard error of the mean of three individual duplications. "*" represents the comparison between the group of control and UV. "\#” represents the comparison to the groups of negative control and blank vector. " $\wedge$ ” represents the comparison between the group of P3 and mP3_1 or mP3_2. All these symbols labeled mean the statistical significance with a $P$-value less than 0.05 by one-way ANOVA.

PCNA-mediated CUL4-DDB1-DTL (Truong and Wu, 2011). DTL and FA pathway are supposed to overlap in DSBs and ICL from replication fork driven by DNA damage but are never studied so far. In our study, the discovery that DTL is enhanced in DNA damage response accompanied with FA pathway activity implies that FA pathway mediated-DNA repair in replication fork may be also regulated by replication licensing factors. The monoubiquitination of FANCD2 is required for FA pathway initiation, and DTL contributes to the recruitment of E3 ubiquitin ligase for the target protein. Although our IP study gives solid evidence for the interaction between DTL and FANCD2, and DTL knockdown substantially causes the reduction of monoubiquitination of FANCD2, it is still hard to determine that the monoubiquitination of FANCD2 is likely to be mediated by DTL. First, CDT1 has a PIP box motif that can be recognized by DTL, but FANCD2 has no such peptide domain. Second, the monoubiquitination of FANCD2 for protein activation and the polyubiquitination for protein degradation are obviously two different regulatory behaviors. FANCD2 so far has never been reported for polyubiquitination. DTL-mediated E3 ligation seems to have nothing to do with FANCD2 monoubiquitination. Nevertheless, our data can determine that DTL has the ability to recruit the activated FANCD2 to DNA lesion, even partially affects the FA pathway activity.

Additionally, p53 is illustrated to bind to the promoter and enhance the transcription of DTL in response to UVinduced DNA damage in ARPE-19 cells. Previous studies have demonstrated that p53 is stabilized and accumulated in the presence of DNA damage by phosphatidylinositol kinase-related kinases ataxia-telangiectasia mutated (ATM), ATM and Rad3-related (ATR), and checkpoint kinase 
(CHK2) (Ou and Schumacher, 2018; Reinhardt and Schumacher, 2012). p53 can facilitate a variety of biological functions in the context of DNA damage. In addition to the cell apoptosis, p53 initiates cell cycle arrest to allow enough time for damage fixing, and p53 has also been implicated in enhancing DNA repair pathways for the DNA lesions clearance (Zhang et al., 2019), including damage-specific DNA binding protein 2 (DDB2), xeroderma pigmentosum complementation group $\mathrm{C}$ and Fanconi anemia complementation group C (FANCC) via transcriptional upregulation (Adimoolam and Ford, 2002; Liebetrau et al., 1997; Scherer et al., 1996). In this study, DTL as a novel p53 target in DNA damage response in RPE is also determined. Interestingly, p53 polyubiquitination for degradation happens to be attributed to the E3 ligase complex of CUL4DDB1-DTL and PCNA (Banks et al., 2006). It is likely a negative feedback regulation to control and maintain the levels of p53 for homeostasis of DNA damage response.

As one of the DNA damage repair systems, FA pathway activity modulated by p53/DTL was conservative for irradiation-mediated DSB damage not only in APRE-19 cells but also in other RPE cells in vitro and in vivo as well as in other cell types. Therefore, our research was supposed to be of benefit to the understanding of the regulatory network responding to DNA damage in multiple human diseases.

In this article, we suggest the role of DTL in FA pathway activation by $\mathrm{p} 53$ in UV-treated RPE, and we believe that this regulation is conserved in other cell types and diseases for studying DNA damage repair.

Availability of Data and Materials: The datasets and supporting materials generated during and/or analysis during the current study are available from the corresponding author on reasonable request.

Author Contribution: All authors participated in the design, interpretation of the studies, analysis of the data, and review of the manuscript. GJ performed all the experiments and analyzed the data. PJ designed the overall project and drafted the manuscript. GQ helped revise the manuscript.

Funding Statement: The authors received no specific funding for this study.

Conflicts of Interest: The authors declare that they have no conflicts of interest to report regarding the present study.

\section{References}

Adimoolam S, Ford JM (2002). p53 and DNA damage-inducible expression of the xeroderma pigmentosum group $\mathrm{C}$ gene. Proceedings of the National Academy of Sciences of the United States of America 99: 12985-12990. DOI 10.1073/ pnas.202485699.

Banks D, Wu M, Higa LA, Gavrilova N, Quan J, Ye T, Kobayashi R, Sun $\mathrm{H}$, Zhang $\mathrm{H}$ (2006). L2DTL/CDT2 and PCNA interact with p53 and regulate $\mathrm{p} 53$ polyubiquitination and protein stability through MDM2 and CUL4A/DDB1 complexes. Cell Cycle 5: 1719-1729. DOI 10.4161/cc.5.15.3150.

Blow JJ, Dutta A (2005). Preventing re-replication of chromosomal DNA. Nature Reviews Molecular Cell Biology 6: 476-486. DOI $10.1038 / \mathrm{nrm} 1663$.
Cheung RS, Castella M, Abeyta A, Gafken PR, Tucker N, Taniguchi T (2017). Ubiquitination-linked phosphorylation of the FANCI S/TQ cluster contributes to activation of the Fanconi anemia I/D2 complex. Cell Reports 19: 2432-2440. DOI 10.1016/j. celrep.2017.05.081.

Federico MB, Vallerga MB, Radl A, Paviolo NS, Bocco JL, Di Giorgio M, Soria G, Gottifredi V, Jinks-Robertson S (2016). Chromosomal integrity after UV irradiation requires FANCD2-mediated repair of double strand breaks. PLoS Genetics 12: e1005792.

Purhonen J, Banerjee R, McDonald AE, Fellman V, Kallijärvi J (2020). JASPAR 2020: update of the open-access database of transcription factor binding profiles. Nucleic Acids Research 48: e87. DOI 10.1093/nar/gkaa516.

Han Y, Chen W, Kuang Y, Sun H, Wang Z, Peng X (2015). UVinduced DNA interstrand cross-linking and direct strand breaks from a new type of binitroimidazole analogue. Chemical Research in Toxicology 28: 919-926. DOI 10.1021/tx500522r.

Jin J, Arias EE, Chen J, Harper JW, Walter JC (2006). A family of diverse Cul4-Ddb1-interacting proteins includes Cdt2, which is required for $S$ phase destruction of the replication factor Cdt1. Molecular Cell 23: 709-721. DOI 10.1016/j. molcel.2006.08.010.

Liebetrau W, Budde A, Savoia A, Grummt F, Hoehn H (1997). p53 activates Fanconi anemia group C gene expression. Human Molecular Genetics 6: 277-283. DOI 10.1093/hmg/6.2.277.

Ou HL, Schumacher B (2018). DNA damage responses and p53 in the aging process. Blood 131: 488-495. DOI 10.1182/blood2017-07-746396.

Panneerselvam J, Wang H, Zhang J, Che R, Yu H, Fei P (2016). BLM promotes the activation of Fanconi Anemia signaling pathway. Oncotarget 7: 32351-32361. DOI 10.18632/oncotarget.8707.

Patton WP, Chakravarthy U, Davies RJ, Archer DB (1999). Comet assay of UV-induced DNA damage in retinal pigment epithelial cells. Investigative Ophthalmology \& Visual Science 40: 3268-3275.

Reinhardt HC, Schumacher B (2012). The p53 network: cellular and systemic DNA damage responses in aging and cancer. Trends in Genetics 28: 128-136. DOI 10.1016/j.tig.2011.12.002.

Roy S (2017). Impact of UV radiation on genome stability and human health, Ultraviolet Light in Human Health. In: Ahmad S (eds.), Diseases and Environment. Advances in Experimental Medicine and Biology, vol. 996. pp. 207-219. Cham: Springer.

Scherer SJ, Welter C, Zang KD, Dooley S (1996). Specific in vitro binding of $\mathrm{p} 53$ to the promoter region of the human mismatch repair gene hMSH2. Biochemical and Biophysical Research Communications 221: 722-728. DOI 10.1006/ bbrc.1996.0663.

Truong LN, Wu X (2011). Prevention of DNA re-replication in eukaryotic cells. Journal of Molecular Cell Biology 3: 13-22. DOI 10.1093/jmcb/mjq052.

Yin K, Chhabra Y, Tropee R, Lim YC, Fane M, Dray E, Sturm RA, Smith AG (2017). NR4A2 promotes DNA double-strand break repair upon exposure to UVR. Molecular Cancer Research 15: 11841196. DOI 10.1158/1541-7786.MCR-17-0002.

Zhang YX, Pan WY, Chen J (2019). p53 and its isoforms in DNA double-stranded break repair. Journal of Zhejiang University -Science B 20: 457-466. DOI 10.1631/jzus.B1900167. 